\title{
Tracing the nonequilibrium topological state of Chern insulators
}

\author{
Michael Schüler and Philipp Werner \\ Department of Physics, University of Fribourg, 1700 Fribourg, Switzerland
}

(Received 30 June 2017; published 16 October 2017)

\begin{abstract}
Chern insulators exhibit fascinating properties, which originate from the topologically nontrivial state characterized by the Chern number. How these properties change if the system is quenched between topologically distinct phases is, however, not fully understood. In this paper, we investigate the quench dynamics of the prototypical massive Dirac model for topological insulators in two dimensions. We consider both dissipationless dynamics and the effect of electron-phonon interactions, and ask how the transient dynamics and nonequilibrium steady states affect simple observables. Specifically, we discuss a time-dependent generalization of the Hall effect and the dichroism of the photoexcitation probability between left and right circularly polarized light. We present optimized schemes based on these observables, which can reveal the evolution of the topological state of the quenched system.
\end{abstract}

DOI: 10.1103/PhysRevB.96.155122

\section{INTRODUCTION}

Topologically nontrivial phases of matter are a subject of intense current research [1,2]. They exhibit a range of intriguing and potentially useful properties, such as the quantum anomalous Hall (QAH) effect. The usual notion is that the intrinsic topology of a system cannot be altered by local perturbations, which results in the protection of certain properties due to time-reversal symmetry. In particular, this effect leads to stable surface or edge states and their extraordinary transport properties.

The relation between the topological phase and corresponding observables is still under active investigation. Originally, such a correspondence has been established in noninteracting systems in equilibrium via the Thouless-KohmotoNightingale-Tijs formula [3]. In this case, the Chern number $\mathcal{C}_{n}$ of each band (labeled by $n$ ) fully characterizes the QAH effect: the Hall conductivity amounts to $\sigma_{x y}=\left(e^{2} / h\right) \sum_{n \in \text { occ }} \mathcal{C}_{n}$. The bulk-insulating system (characterized by $\sigma_{x x}=0$ and $\sigma_{x y} \neq 0$ ) is then called a Chern or quantum Hall insulator (QHI). The Chern number, on the other hand, is obtained via the Berry curvature from the wave function $\left|\phi_{\mathbf{k} n}\right\rangle$ directly-a quantity that is, strictly speaking, available for noninteracting systems (or within mean-field treatments) only. A possible extension in the context of many-body perturbation theory can be obtained by constructing an effective Hamiltonian involving the self-energy at zero frequency [4], which allows us to study the interplay of topological properties and correlation effects in QHIs [5-7]. This approach is based on the connection of the Chern number to the winding number [8]. Alternatively, topological states can be classified by studying the response of a system to external gauge fields [9]. While these approaches work for noninteracting as well as for interacting electrons, the underlying assumption is that the system is in its ground state. Hence the established concepts are not necessarily applicable to finite temperature or nonequilibrium scenarios, which involve excited states.

This is particularly true for global perturbations such as quenches of the Hamiltonian parameters. For instance, a straightforward definition of a time-dependent Chern number $\mathcal{C}_{n}(t)$ from the time-evolving wave functions $\left|\phi_{\mathbf{k} n}(t)\right\rangle$ of a noninteracting system will remain constant under unitary evolution. On the other hand, the Hall conductivity $\sigma_{x y}$ can change, e.g., after a quench, which means that it is generally not identical to the Chern number (up to the prefactor $e^{2} / h$ ), in contrast to the equilibrium case [10-12]. Similarly, the bulk-boundary correspondence might be lost after a quench $[13,14]$. It is thus a relevant task to identify experimentally accessible quantities, which allow us to trace the nonequilibrium evolution of topologically nontrivial systems.

In this paper, we investigate different schemes that enable us to study the nonequilibrium and transient dynamics of QHIs. We focus on (i) the time-resolved Hall effect using appropriately shaped electromagnetic pulses, and (ii) the photoabsorption asymmetry with respect to left/right circularly polarized light - a novel approach, which has been suggested recently [15]. Both methods are based on directly observable quantities and are thus well suited for the study of (effectively) noninteracting as well as correlated and/or dissipative systems. We demonstrate their applicability by considering as a generic example the well-known massive Dirac model (MDM) on a square lattice [16], which captures [17] the topological phase transition in HgTe quantum wells [18]. We focus on quench dynamics and demonstrate how a transition between phases of distinct topological character manifests itself in these observables. Furthermore, we study the influence of dissipation due to electron-phonon (EL-PH) coupling on the transient dynamics to demonstrate the robustness of the proposed schemes.

\section{MODEL}

As a paradigm model for two-dimensional systems we consider the massive Dirac model on a square lattice. The electronic Hamiltonian reads

$$
\hat{H}_{\mathrm{el}}=\sum_{\mathbf{k} \in \mathrm{BZ}} \sum_{a b}\left[h_{\mathrm{el}}(\mathbf{k})\right]_{a b} \hat{c}_{\mathbf{k} a}^{\dagger} \hat{c}_{\mathbf{k} b},
$$

where the k-dependent single-particle Hamiltonian $\left[h_{\mathrm{el}}(\mathbf{k})\right]_{a b}=\left\langle\mathbf{k} a\left|\hat{h}_{\mathrm{el}}(\mathbf{k})\right| \mathbf{k} b\right\rangle$ has the generic form $\hat{h}_{\mathrm{el}}(\mathbf{k})=\sum_{\alpha=x, y, z} d_{\alpha}(\mathbf{k}) \hat{\sigma}^{\alpha}$. Here, the $\hat{\sigma}^{\alpha}$ denote the 
pseudospin operators with respect to the underlying bands. The coefficients $d_{\alpha}(\mathbf{k})$ are defined by

$$
\begin{aligned}
& d_{x}(\mathbf{k})=\lambda \sin \left(k_{x} a\right), d_{y}(\mathbf{k})=\lambda \sin \left(k_{y} a\right), \\
& d_{z}(\mathbf{k})=-2 T_{0}\left[\cos \left(k_{x} a\right)+\cos \left(k_{y} a\right)\right]-M .
\end{aligned}
$$

The eigenstates of the single-particle Hamiltonian are denoted by $\hat{h}_{\mathrm{el}}(\mathbf{k})\left|\phi_{\mathbf{k} n}\right\rangle=\varepsilon_{n}(\mathbf{k})\left|\phi_{\mathbf{k} n}\right\rangle$.

Note that we limit ourselves to a spin-restricted model here, as the Hamiltonian (1) is spin independent. As shown in Ref. [17], in HgTe quantum wells, which are well modeled by Eqs. (1) and (2), doping with manganese allows us to shift the spin-up/spin-down bands in such a way that only one spin channel remains important. Here we assume such a situation and thus focus on the charge QAH effect instead of the usual quantum spin Hall effect (QSH).

It is straightforward to see that diagonalizing the Hamiltonian (1) gives rise to a trivial band insulator (BI) for $M<-4\left|T_{0}\right|$, while the system corresponds to a topological insulator $(\mathrm{TI})^{1}$ for $0>M>-4\left|T_{0}\right|$. The topological character of the bands can be determined by the Chern number $\mathcal{C}_{n}$, which is defined (for each band $n=1,2$, respectively) by the integral over the Berry curvature

$$
\mathcal{C}_{n}=\frac{1}{2 \pi} \int d \mathbf{k}\left(\frac{\partial A_{y}^{(n)}}{\partial k_{x}}-\frac{\partial A_{x}^{(n)}}{\partial k_{y}}\right) .
$$

Here, $\mathbf{A}^{(n)}(\mathbf{k})=-i\left\langle\phi_{\mathbf{k} n} \mid \nabla_{\mathbf{k}} \phi_{\mathbf{k} n}\right\rangle$ is the Berry connection corresponding to the upper $(n=1)$ or lower $(n=2)$ band.

\section{A. Quench dynamics}

A quench from the BI into the TI phase (or vice versa) offers insight into the underlying topological properties. For instance, the insulating state with nontrivial Chern number cannot be altered under unitary time evolution (which preserves time-reversal symmetry), hence, the question arises as to which state the system is driven. Furthermore, quenches may induce dynamical phase transitions, which have recently been studied for the MDM [19]. Quenches can be realized, for instance, by photodoping pulses in interacting electronic systems, leading to transient band shifts [20,21], or by changing the strength of the periodic driving in Floquet topological insulators $[22,23]$.

Here we study quenches of the gap parameter $M$ between the values $M_{\mathrm{TI}}=-3\left|T_{0}\right|$ and $M_{\mathrm{BI}}=-5\left|T_{0}\right|$. The band hybridization is fixed at $\lambda=0.2\left|T_{0}\right|$ in what follows. The transition from phase $\mathrm{A}\left(M=M_{\mathrm{TI} / \mathrm{BI}}\right)$ to $\mathrm{B}$ $\left(M=M_{\mathrm{BI} / \mathrm{TI}}\right)$ is triggered by a softened ramp of the form

$$
\hat{H}_{\mathrm{el}}(t)=[1-\alpha(t)] \hat{H}_{\mathrm{el}}^{\mathrm{A}}+\alpha(t) \hat{H}_{\mathrm{el}}^{\mathrm{B}},
$$

with $\alpha(t)=1-\cos \left[\pi\left(t-t_{\mathrm{q}}\right) / T_{\mathrm{q}}\right]$, defined by the quench time $t_{\mathrm{q}}$ and the duration $T_{\mathrm{q}}\left(t_{\mathrm{q}}<t<t_{\mathrm{q}}+T_{\mathrm{q}}\right)$.

\footnotetext{
${ }^{1}$ Even though the term topological insulator (TI) refers to a more general concept than the QAH insulator, we use the abbreviation TI throughout the text.
}

In the absence of any further interactions, the dynamics can be captured by solving the time-dependent Schrödinger equation

$$
i \frac{d}{d t}\left|\psi_{\mathbf{k} n}(t)\right\rangle=\hat{h}_{\mathrm{el}}(t)\left|\psi_{\mathbf{k} n}(t)\right\rangle,\left|\psi_{\mathbf{k} n}(t=0)\right\rangle=\delta_{n, 1}\left|\phi_{\mathbf{k} 1}\right\rangle,
$$

where we have assumed half-filling. The time-dependent single-particle Hamiltonian $\hat{h}_{\mathrm{el}}(t)$ is defined in analogy to Eq. (4). As mentioned above, a straightforward generalization of the definition of the Chern number (3) as

$$
\mathcal{C}_{n}(t)=\frac{1}{2 \pi} \int d \mathbf{k}\left(\frac{\partial A_{y}^{(n)}(t)}{\partial k_{x}}-\frac{\partial A_{x}^{(n)}(t)}{\partial k_{y}}\right)
$$

with the time-dependent Berry connection $\mathbf{A}^{(n)}(\mathbf{k}, t)=$ $-i\left\langle\psi_{\mathbf{k} n}(t) \mid \nabla_{\mathbf{k}} \psi_{\mathbf{k} n}(t)\right\rangle$ will be invariant under any unitary time evolution [10,14]. As discussed by Wang et al. (Ref. [11]), the Hall conductance may, however, undergo a change. Assuming that decoherence effects have suppressed the off-diagonal elements of the density matrix after sufficiently long time, the steady-state Hall conductance can be defined as

$$
\sigma_{x y}=\frac{e^{2}}{2 \pi h} \sum_{n} \int d \mathbf{k} f_{n}(\mathbf{k})\left(\frac{\partial A_{y}^{(n), \mathrm{B}}}{\partial k_{x}}-\frac{\partial A_{x}^{(n), \mathrm{B}}}{\partial k_{y}}\right) .
$$

Here, $\mathbf{A}^{(n), \mathrm{B}}(\mathbf{k})$ stands for the Berry connection of the postquench Hamiltonian $\hat{h}_{\mathrm{el}}^{\mathrm{B}}$, whereas $f_{n}(\mathbf{k})$ is the occupation with respect to the postquench band structure. For the two-band MDM, $f_{n}(\mathbf{k})$ is easily expressed in terms of the overlap of the prequench and postquench basis and does not depend on the quench details.

\section{B. Electron-phonon coupling}

The nonequilibrium Hall conductance (7) assumes that the system has lost the coherences due to environmental coupling; otherwise, the coherent oscillations after excitations induced by the quench hamper a unambiguous definition of $\sigma_{x y}$. The most important intrinsic source for such dephasing effects in real systems is (besides structural defects) EL-PH coupling.

As demonstrated in Ref. [24], the MDM approaches the respective ground state after an quench if the coupling to the environment is dominated by dephasing. However, the coupling to the lattice vibrations entails dissipative population dynamics, as well. Therefore, the resulting steady state is, in general, different from the quench calculation, which considers dephasing only [25]. This has been shown, for instance, in Floquet topological insulators subject to electron-phonon coupling [26], where the out-of-equilibrium Hall conductance differs from the TKNN relation [27]. In the current study, we treat the EL-PH coupling explicitly by extending the Hamiltonian to

$$
\hat{H}(t)=\hat{H}_{\mathrm{el}}(t)+\hat{H}_{\mathrm{el}-\mathrm{ph}}+\hat{H}_{\mathrm{ph}} .
$$

For the interaction term, we use the Fröhlich coupling [28] in two dimensions:

$$
\hat{H}_{\mathrm{el}-\mathrm{ph}}=\frac{\gamma}{\sqrt{N_{k}}} \sum_{\mu \mathbf{q}} \mathbf{u}_{\mu \mathbf{q}} \cdot \frac{\mathbf{q}}{q} \sum_{\mathbf{k}} \hat{c}_{\mathbf{k} n}^{\dagger} \hat{c}_{\mathbf{k}-\mathbf{q} n} \hat{Q}_{\mu \mathbf{q}} \cdot
$$

Here, $\gamma$ is a constant determining the overall coupling strength and $N_{k}$ denotes the number of $\mathbf{k}$ points. The phonon modes 

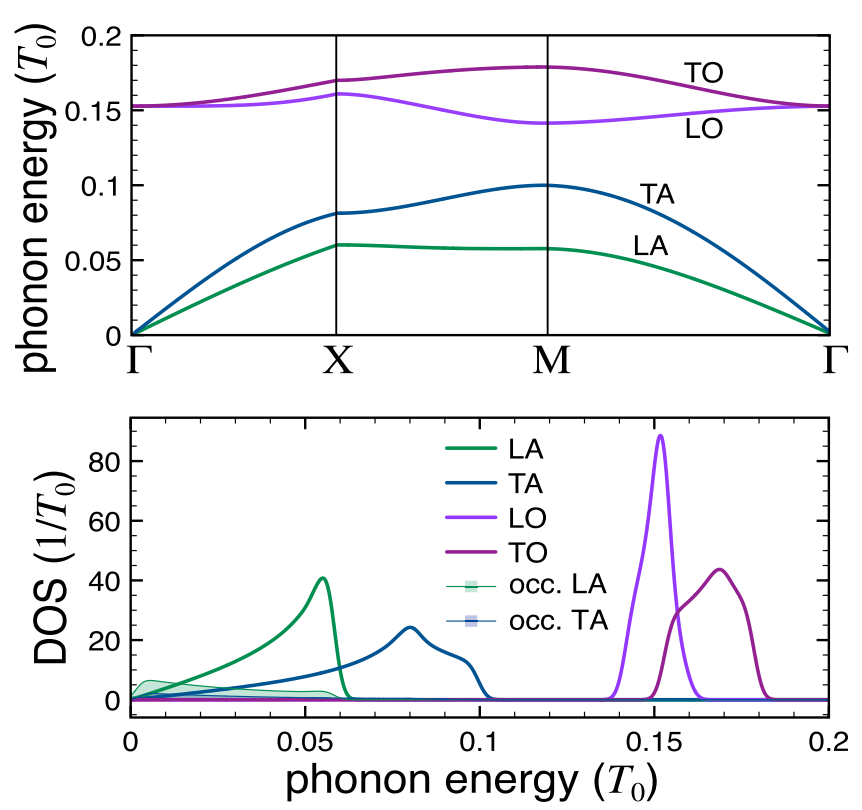

FIG. 1. Top: Dispersion of the longitudinal acoustic (LA), transverse acoustic (TA), longitudinal optical (LO), and transverse optical (TO) phonon modes. The energy is measured in units of the hopping constant $T_{0}$. Bottom: Corresponding density of states (DOS) along with the occupation-weighted DOS for the LA and TA modes (filled curves).

$\mu$ with momentum $\mathbf{q}$ are represented by the corresponding mode vector $\mathbf{u}_{\mu \mathbf{q}}$ and the coordinate (momentum) operators $\hat{Q}_{\mu \mathbf{q}}\left(\hat{P}_{\mu \mathbf{q}}\right)$. The latter define the phonon Hamiltonian by

$$
\hat{H}_{\mathrm{ph}}=\sum_{\mu \mathbf{q}} \frac{\omega_{\mu}(\mathbf{q})}{2}\left(\hat{P}_{\mu \mathbf{q}}^{\dagger} \hat{P}_{\mu \mathbf{q}}+\hat{Q}_{\mu \mathbf{q}}^{\dagger} \hat{Q}_{\mu \mathbf{q}}\right) .
$$

To compute the phonon modes in the square lattice, we assume a diatomic basis characterized by masses $M_{1,2}$ and three force constants $c_{1,2,3}$ describing the nearest-neighbor, next-nearest-neighbor, and diagonal interaction, respectively. Diagonalizing the dynamical matrix yields two acoustic and two optical (longitudinal and transverse) phonon modes. The parameters are chosen to produce a similar phonon dispersion $\omega_{\mu}(\mathbf{q})$ as is known for $\mathrm{HgTe}$ [29]—up to an overall scaling constant. We have slightly increased the phonon energies to make the effects due to the EL-PH coupling more visible. However, it is important to note that we stay in the realistic parameter regime where only intraband transitions can be induced by scattering from phonons. The phonon dispersion and the corresponding density of states (DOS) is shown in Fig. 1. In what follows, we assume low temperatures by fixing the inverse temperature at $\beta=40\left|T_{0}\right|^{-1}$, such that only the acoustic phonons around the $\Gamma$ point are thermally activated.

It should be mentioned that the role of the EL-PH coupling in topological insulators, in particular at the surface, is a topic of recent discussions. While some works point out the strong influence of EL-PH coupling for inelastic scattering [30-32] and for intraband relaxation of photoexcited systems [33], other measurements suggest weak EL-PH interaction effects [34]. Here, we take a different angle and treat the coupling strength $\gamma$ as a parameter.

\section{Equations of motion in the presence of electron-phonon interactions}

The full Hamiltonian (8) constitutes an interacting electronboson model. The numerically exact solution is out of reach for the typical number of points in reciprocal space. Furthermore, since the phonon energies $\omega_{\mu}(\mathbf{q})$ are much smaller than the electronic energy scale, a weak-coupling treatment is suitable. In this context, the nonequilibrium Green's function (NEGF) approach in its time-dependent formulation has become an important tool recently [35-41]. However, most of the approaches resort to a local approximation to the EL-PH interaction. This approximation excludes intraband transitions, which are, as discussed above, the only available relaxation channel in our case. Therefore, a momentum-dependent treatment of the phonons is required, which increases the computational costs of the NEGF method considerably. Since neither renormalization effects of the electronic structure due to the coupling to the phonons nor the back action on the phononic degrees of freedom is of particular significance for the relaxation in the MDM, one can employ a simplified theory based on a master-equation approach within the Markovian approximation [42]. The usual Lindblad equation, however, is formulated in terms of the many-body density matrix [43]. For calculating the time evolution of the single-particle density matrix, $\rho_{i j}(\mathbf{k} ; t)=\left\langle\hat{c}_{\mathbf{k} i}^{\dagger}(t) \hat{c}_{\mathbf{k} j}(t)\right\rangle$, additional conditions are required, reflecting not only the overall particle conservation, but the fermionic statistics, as well. This can be achieved by extending the linear Lindblad equation to a nonlinear master equation [44,45]. Alternatively, equivalent master equations can also be obtained from with NEGF formulation within the generalized Kadanoff-Baym ansatz [46] and applying the Markovian approximation [47].

Within the master-equation approach, the equation of motion (EOM) for the single-particle density matrix reads

$$
\frac{d}{d t} \boldsymbol{\rho}(\mathbf{k} ; t)=-i\left[\mathbf{h}_{\mathrm{el}}(\mathbf{k} ; t), \boldsymbol{\rho}(\mathbf{k} ; t)\right]+\mathbf{I}(\mathbf{k} ; t),
$$

where we have employed a more compact matrix notation. Besides the unitary time evolution captured by the first term, the EL-PH coupling is described by the scattering term

$$
\begin{aligned}
I_{i j}(\mathbf{k} ; t)= & \frac{1}{2} \sum_{\mu \mathbf{q}} \sum_{k l m}\left[\bar{\rho}_{i k}(\mathbf{k} ; t) \mathcal{R}_{k l, j m}^{\mu \mathbf{q}}(\mathbf{k}, \mathbf{k}-\mathbf{q}) \rho_{l m}(\mathbf{k}-\mathbf{q} ; t)\right. \\
& \left.-\bar{\rho}_{k l}(\mathbf{k}+\mathbf{q} ; t) \mathcal{R}_{k i, l m}^{\mu \mathbf{q}}(\mathbf{k}+\mathbf{q}, \mathbf{k}) \rho_{m j}(\mathbf{k}, t)\right] .
\end{aligned}
$$

Here, $\bar{\rho}_{i j}(\mathbf{k} ; t)=\delta_{i j}-\rho_{i j}(\mathbf{k} ; t)$ is the density matrix of the hole states, while $\mathcal{R}_{k l, j m}^{\mu \mathbf{q}}\left(\mathbf{k}_{1}, \mathbf{k}_{2}\right)$ denotes the scattering rate, which determines the transition probability. For the derivation of the master equation (11)-(12) we followed Ref. [44] and split the indices into band indices and quasimomenta. Exploiting the momentum conservation built into the EL-PH coupling matrix elements, the scattering term can be readily formulated in terms of electron (k) and phonon (q) momenta. As the scattering events are limited to intraband transitions governed by momentum (and energy) conservation, the scattering rates simplify to [45]

$$
\mathcal{R}_{k l, j m}^{\mu \mathbf{q}}\left(\mathbf{k}_{1}, \mathbf{k}_{2}\right)=\delta_{\mathbf{k}_{1}, \mathbf{k}_{2}+\mathbf{q}} \delta_{k j} \delta_{l m} R_{j l}^{\mu \mathbf{q}}\left(\mathbf{k}_{1}, \mathbf{k}_{2}\right)
$$


with

$$
R_{j l}^{\mu \mathbf{q}}\left(\mathbf{k}_{1}, \mathbf{k}_{2}\right)=\frac{\gamma^{2}}{N_{k}} \frac{1}{q^{2}}\left|\mathbf{u}_{\mu \mathbf{q}} \cdot \mathbf{q}\right|^{2} D_{\mu \mathbf{q}}^{>}\left[\varepsilon_{l}\left(\mathbf{k}_{2}\right)-\varepsilon_{j}\left(\mathbf{k}_{1}\right)\right] .
$$

Here, the spectral function of the unoccupied phonon modes (greater Green's function in the NEGF context) is defined by

$$
\begin{aligned}
D_{\mu \mathbf{q}}^{>}(\omega)= & 2 \pi\left[N_{B}(\omega)+1\right]\left\{\delta\left[\omega-\omega_{\mu}(\mathbf{q})\right]\right. \\
& \left.-\delta\left[\omega+\omega_{\mu}(\mathbf{q})\right]\right\},
\end{aligned}
$$

where $N_{B}(\omega)$ denotes the Bose distribution. In practice, the Dirac- $\delta$ functions in Eq. (15) are replaced by Gaussians with a small broadening parameter $\eta$.

In principle, the validity of the master EOM (11) is limited to scenarios without external driving due to the energy conservation implicit in the scattering term (14). However, the time-dependent perturbations employed here are fast compared to the time scale of the EL-PH scattering: (i) the gap parameter $M$ is ramped within a time interval of $T_{\mathrm{q}}=5.0$; (ii) the duration of the circularly polarized laser pulses is set to $T_{\mathrm{p}}=5.0$. Therefore, the driven dynamics is dominated by the unitary dynamics, whereas dissipation effects manifest themselves at a later stage of the time evolution, where energy conservation holds.

Note that the definition of the scattering terms (12)-(14) depends on the electronic eigenenergies explicitly. The EOM (11) thus has to be solved in the eigenbasis of either the prequench or the postquench Hamiltonian. Since we employ a ramped quench scheme here, we have chosen the prequench Hamiltonian $\mathbf{h}_{\mathrm{el}}\left(\mathbf{k} ; t_{\mathrm{q}}\right)$ as the reference point and inserted the corresponding electronic energies in Eq. (14). On the other hand, in a sudden quench scenario it is natural to choose the postquench basis and insert the prequench density matrix as initial condition.

For each momentum $\mathbf{k}$ in the Brillouin zone, the EOM (11) constitutes a nonlinear ordinary differential equation. For its solution we split off the unitary time evolution and treat it using exact matrix exponentials. In this way, the quench or driving dynamics is treated accurately (and, when the Hamiltonian is time independent, exactly). For the higher-order treatment of the scattering term we have adopted a method similar to the one discussed in Ref. [48]. The full numerical scheme is detailed in the Appendix.

\section{OBSERVABLES IN EQUILIBRIUM}

Let us now proceed by defining the observables, which will be used to trace the nonequilibrium dynamics. We first consider the equilibrium case, where the system is either in the band insulating or QHI phase and then discuss how to extend the schemes to the time-dependent case.

\section{A. Circular asymmetry}

As suggested by Tran et al. [15], the depletion rate $\Gamma_{n}^{(+)}(\omega)$ $\left[\Gamma_{n}^{(-)}(\omega)\right]$ upon irradiation of left (right) circularly polarized light with frequency $\omega$ yields a direct measure of the Chern number of band $n$. In particular, the frequency-integrated asymmetry signal $\int d \omega\left[\Gamma_{n}^{(+)}(\omega)-\Gamma_{n}^{(-)}(\omega)\right]$ is proportional to $\mathcal{C}_{n}$. This property was derived for a noninteracting system; however, it is generic and can also be exploited in interacting
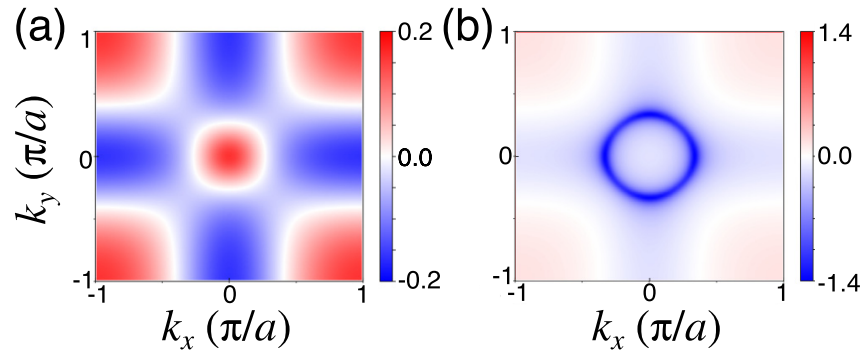

FIG. 2. Circular asymmetry $\Delta(\mathbf{k})$ of the transition probability for (a) the $\mathrm{BI}\left(M=M_{\mathrm{BI}}=-5\left|T_{0}\right|\right)$ and (b) the $\mathrm{TI}\left(M=M_{\mathrm{TI}}=-3\left|T_{0}\right|\right)$.

systems. A closely related effect is the pronounced polarization dependence observed in angle-resolved photoemission (ARPES) from graphene, mapping out the Berry phase of the individual bands $[49,50]$. Alternative ways of extracting the topological character of the system in experiments, besides the aforementioned Hall effect, are Aharonov-Bohm-type interferometry [51] in optical traps and spin-polarized measurements [52]. However, here we focus on observables that can be easily extended to the transient regime.

For the two-band MDM, Fermi's golden rule yields

$$
\Gamma_{1}^{( \pm)}(\omega) \propto \sum_{\mathbf{k}}\left|\left\langle\phi_{\mathbf{k} 2}\left|\hat{V}^{( \pm)}(\mathbf{k})\right| \phi_{\mathbf{k} 1}\right\rangle\right|^{2} \delta\left(\varepsilon_{2}(\mathbf{k})-\varepsilon_{1}(\mathbf{k})-\omega\right) .
$$

Here, the transition operator, derived from the standard Peierls substitution, reads

$$
\hat{V}^{( \pm)}(\mathbf{k})=\frac{\partial \hat{h}_{\mathrm{el}}(\mathbf{k})}{\partial k_{x}} \pm i \frac{\partial \hat{h}_{\mathrm{el}}(\mathbf{k})}{\partial k_{y}} .
$$

Provided the transition is permitted by energy conservation, the excitation probability is determined by the matrix elements $D^{( \pm)}(\mathbf{k})=\left|\left\langle\phi_{\mathbf{k} 2}\left|\hat{V}^{( \pm)}(\mathbf{k})\right| \phi_{\mathbf{k} 1}\right\rangle\right|^{2}$. The asymmetry $\Delta(\mathbf{k})=D^{(+)}(\mathbf{k})-D^{(-)}(\mathbf{k})$ is presented for the BI and the TI in Figs. 2(a) and 2(b), respectively. We neglect the EL-PH coupling at this point $(\gamma=0)$.

As can be seen in Fig. 2, the main difference between BI and TI is the strong negative asymmetry in the vicinity of the $\Gamma$ point in the latter case [Fig. 2(b)], which is most pronounced at the $\mathbf{k}$ points where the avoided crossing between the two bands occurs. This can be understood from the fact that $\Delta(\mathbf{k})$ is proportional to the Berry curvature in the two-band case [15].

In view of transient measurements, it would be most useful to extract the topological character of the system by applying short (circularly polarized) pulses rather than the continuouswave (plus integrating over frequencies) approach presented in Ref. [15]. Figure 2 suggests to consider transitions close to the $\Gamma$ point, where the difference in the Berry curvature between the BI and TI is most pronounced. The thus required spectral resolution has to be balanced against time resolution determined by the pulse duration. We have chosen electric field pulses of the form

$$
\mathbf{E}^{( \pm)}(t)=F_{0} \sin ^{2}\left(\frac{\pi\left(t-t_{0}\right)}{T_{\mathrm{p}}}\right) \operatorname{Re}\left\{e^{-i \omega_{0}\left(t-t_{0}\right)} \boldsymbol{\epsilon}^{( \pm)}\right\}
$$

for $t_{0}<t<t_{0}+T_{\mathrm{p}}$. The left or right polarization vector (superscript + or - , respectively) is given by $\boldsymbol{\epsilon}^{( \pm)}=\mathbf{e}_{x} \pm i \mathbf{e}_{y}$. 


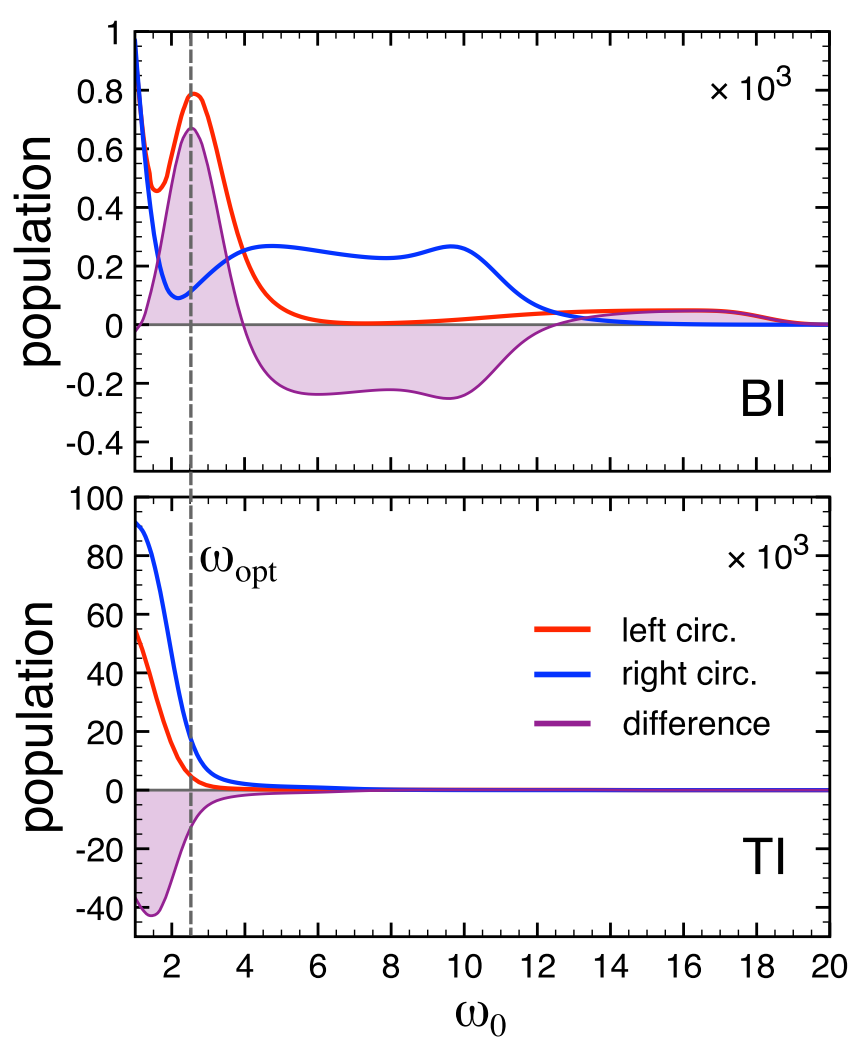

FIG. 3. Population of the upper band after applying a pulse with central frequency $\omega_{0}$ to the BI (top) and TI (bottom). The dashed line marks the optimal pulse frequency $\omega_{\text {opt }}$ where the difference between the $\mathrm{BI}$ and $\mathrm{TI}$ response is most pronounced.

In order to determine the corresponding asymmetry signal, we propagate the time-dependent Schrödinger equation in the presence of the electric field (18) by the Peierls substitution $\hat{h}_{\mathrm{el}}^{( \pm)}(\mathbf{k} ; t) \rightarrow \hat{h}_{\mathrm{el}}^{( \pm)}\left[\mathbf{k}-\mathbf{A}^{( \pm)}(t) ; t\right]\left[\mathbf{A}^{( \pm)}(t)\right.$ is the vector potential corresponding to the field] in the weak-field limit $\left(F_{0}=0.5\right)$. The expected left-right asymmetry of the depletion rate translates into an asymmetry of the photoexcitation probability. After some tests we found that pulses as short as $T_{\mathrm{p}}=5.0$ offer a good comprise between sharpness in frequency space and pulse duration. The excitation probability for both the BI and the TI case is depicted in Fig. 3 as a function of the central frequency $\omega_{0}$.

In line with Ref. [15], integrating over all frequencies yields zero for the $\mathrm{BI}$ (since $\mathcal{C}_{1}=0$ ), while a nonzero value is obtained for the TI. As expected from the behavior of the matrix elements (see Fig. 2), the region in reciprocal space where the Berry curvature is the strongest in the TI case is particularly suited for mapping out the topological character of the system. This leads to the optimal frequency $\omega_{\text {opt }} \simeq 2.5$ where the BI predominantly absorbs left circularly polarized light [corresponding to the red region around the origin in Fig. 2(a)], but where the Berry curvature leads to a strongly enhanced absorption of right circularly polarized radiation in the TI case. Choosing $\omega_{0}=\omega_{\text {opt }}$ we obtain a field pulse, which is ideally suited for tracing the transient dynamics of the system upon photoexcitation or after a quench. Note that the absorbance of the $\mathrm{BI}$ is reduced compared to the TI due to the larger band gap.

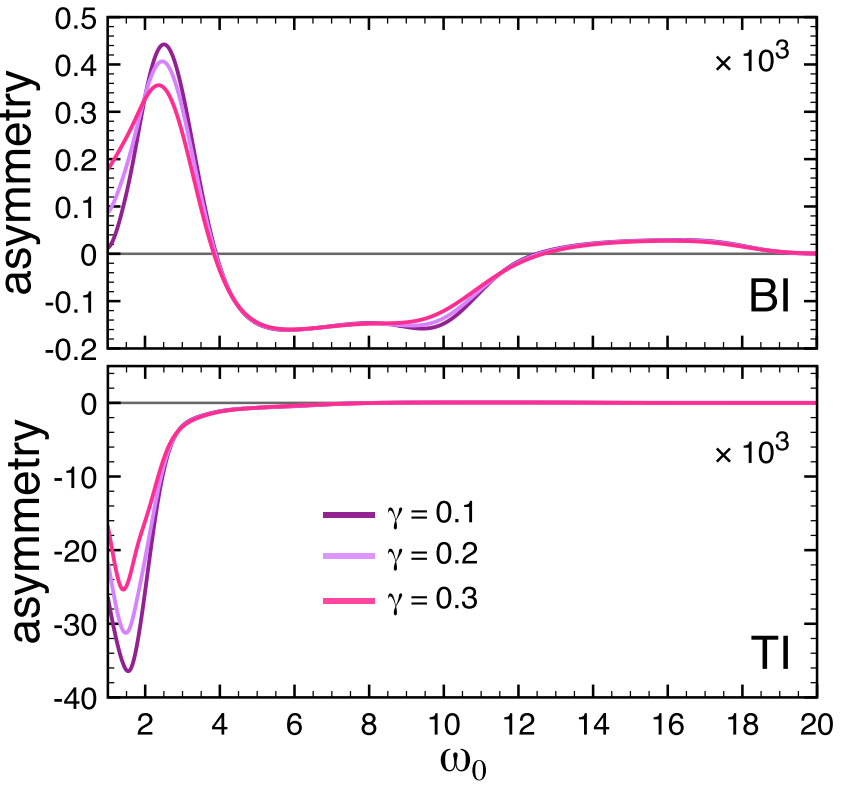

FIG. 4. Asymmetry signal analogous to Fig. 3, but for different EL-PH coupling strengths $\gamma>0$.

The next important question to address is if a similar behavior can be expected in the presence of EL-PH coupling. To address this issue, we solved the master equation (11) including the electromagnetic field and computed, as for the noninteracting case, the photoexcitation probability. The result for several moderate coupling strengths $\gamma$ is presented in Fig. 4. Besides an overall suppression of the absorbance and the less pronounced difference between the the excitation probabilities for left/right circularly polarized light, the qualitative behavior is consistent with the dissipationless case (Fig. 3). The visible difference of the asymmetry for the relatively strong EL-PH couplings demonstrated in Fig. 4 implies that one can obtain valuable information on the topological character even for weak to moderate strength of dissipative effects.

\section{B. Time-resolved Hall effect}

The emergence of the integer Hall effect $\sigma_{x y}=\left(e^{2} / h\right) \mathcal{C}_{1}$ provides direct access to the topological character in equilibrium. A possible extension of this concept to a time-dependent scenario is-similarly as in Sec. III A-to apply suitably shaped pulses with parameters optimized in the equilibrium case. To this end, we computed the optical conductivity $\sigma_{\alpha \beta}(\omega)$ for both the BI and the TI phase. The real part is shown in Fig. 5 .

The plateau in $\sigma_{x y}(\omega)$ at small frequencies gives us some guidance in choosing the spectral features of a suitable probe pulse $\mathbf{E}(t)$, which allows us to map out the topological character: (i) the pulse needs to be short to enable us to trace the transient dynamics; (ii) the pulse in frequency space needs to have a maximal overlap with the region $\omega \approx 0$; and (iii) $\int d t \mathbf{E}(t)=0$ is required within the dipole approximation. Electromagnetic field pulses, which optimally fulfill the criteria (i)-(iii) are half-cycle pulses (HCPs) [53]. HCPs are pulses with a dominant, short peak and a weak and long tail (which hardly influences the dynamics). The dominant peak makes the field effectively unipolar, as the 

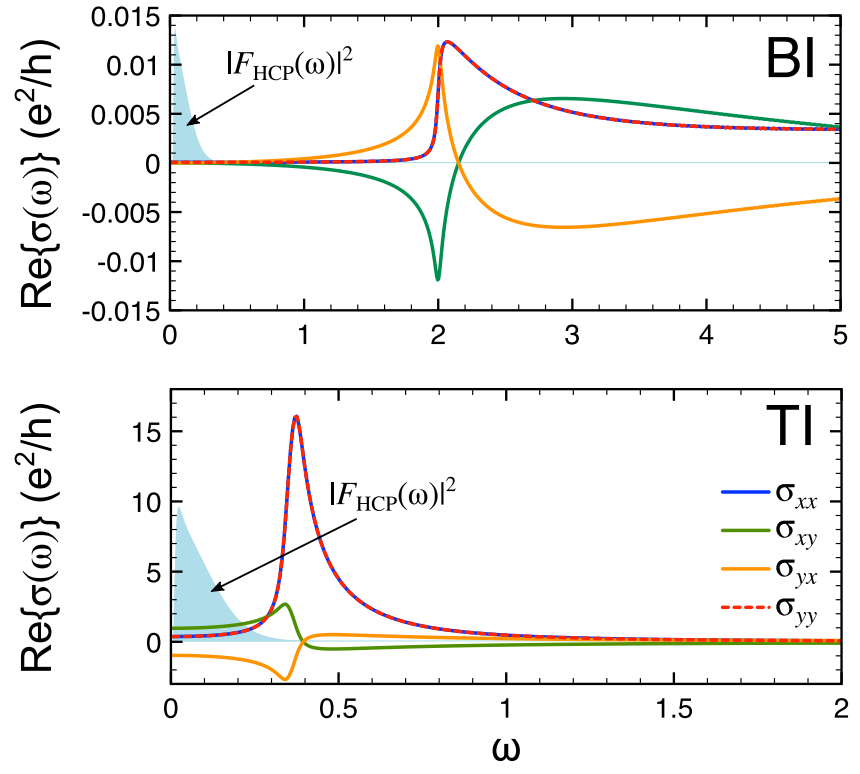

FIG. 5. Real part of the optical conductivity for the BI (top) and the TI (bottom). The filled curve illustrates the power spectrum of the HCP used for the time-dependent calculations (see text). The small but finite value of $\sigma_{x x}(\omega=0)$ is due a small broadening of the Fermi surface.

spectral weight is maximal in the vicinity of $\omega \approx 0$. Here we employ the parametrization $\mathbf{E}_{\mathrm{HCP}}(t)=E_{0} \boldsymbol{\epsilon} F_{\mathrm{HCP}}\left(t-t_{0}\right)$ with

$$
F_{\mathrm{HCP}}(t)=x\left(e^{-x^{2} / 2}-\frac{1}{b^{2}} e^{-x / b}\right), x=\frac{t}{T_{\mathrm{p}}} .
$$

The parameters are the effective pulse duration $T_{\mathrm{p}}$ and the shape parameter $b$, which we fix at $b=8$ in accordance [54] with typical pulses generated in experiments $[55,56]$. The analytical expression in Eq. (19) fulfils $\int_{0}^{\infty} d t F_{\mathrm{HCP}}(t)=0$ exactly. The corresponding power spectrum in frequency space is shown in Fig. 5. We have found $T_{\mathrm{p}}=10$ to be a good compromise between a short pulse duration and maximal overlap with the plateau region of the TI, as can be seen in Fig. 5.

The properties of the HCPs translate-in combination with the frequency dependence of the optical conductivity—into a distinct behavior of the time-dependent current

$$
J_{\alpha}(t)=\sum_{\mathbf{k}} \operatorname{Tr}\left[\mathbf{v}^{\alpha}(\mathbf{k}-\mathbf{A}(t)) \rho(\mathbf{k} ; t)\right] .
$$

Here, $\mathbf{v}^{\alpha}(\mathbf{k})=\partial \mathbf{h}_{\mathrm{el}}(\mathbf{k}) / \partial k_{\alpha}$ is the velocity matrix. Within the weak-field regime, linear response theory applies and relates the current to the driving field and the optical conductivity:

$$
J_{\alpha}(t)=E_{0} \sum_{\beta=x, y} \int_{0}^{t} d t^{\prime} \sigma_{\alpha \beta}\left(t-t^{\prime}\right) \epsilon_{\beta} F_{\mathrm{HCP}}\left(t^{\prime}-t_{0}\right) .
$$

Assuming a linearly polarized HCP in the $x$ direction, the linear response relation (21) yields for the current in the $y$ direction in frequency space

$$
J_{y}(\omega)=E_{0} \sigma_{y x}(\omega) F_{\mathrm{HCP}}(\omega) \simeq E_{0} \sigma_{y x}(\omega=0) F_{\mathrm{HCP}}(\omega),
$$
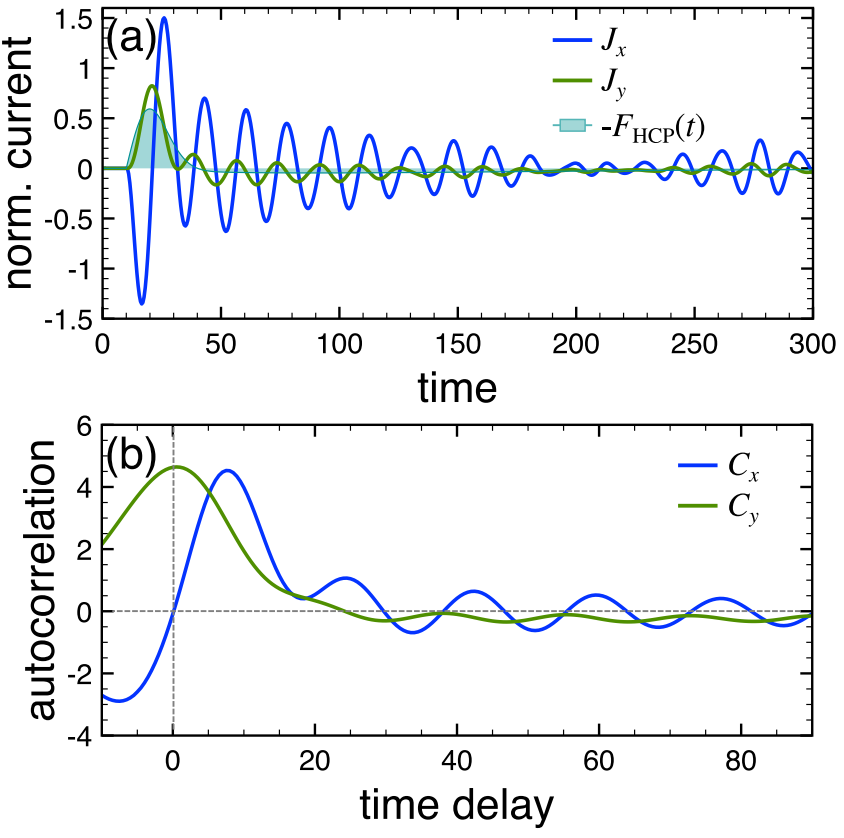

FIG. 6. (a) Time-dependent current $J_{\alpha}(t)$ induced by a HCP with $T_{\mathrm{p}}=10, t_{0}=10$, and $E_{0}=10^{-4}$, polarized in the $x$ direction. The current has been normalized by the pulse strength. (b) Corresponding pulse-current correlation functions [Eq. (24)] as functions of the delay $\Delta t$.

which implies for the time-dependent current

$$
J_{y}(t) \simeq E_{0} \sigma_{y x}(\omega=0) F_{\mathrm{HCP}}(t)
$$

Thus, the current orthogonal to the field polarization is expected to closely resemble the driving pulse in the TI case, while the current will almost vanish for the BI. This behavior is confirmed by the numerical solution of the Schrödinger equation in the presence of the HCP (19) and the resulting time-dependent current $J_{\alpha}(t), \alpha=x, y$ displayed in Fig. 6 for the TI case. The Hall current $J_{y}(t)$ has a strong peak for times where the HCP has its maximum, while the current $J_{x}(t)$ shows an oscillatory behavior. As expected, the simulations show that the Hall current is negligible for the BI phase (not shown).

Since detecting a time-dependent current on the typical time scale of the pulse (which is in the femtosecond to picosecond range) is difficult in experiments, we propose to analyze the behavior of the pulse-current correlation function

$$
C_{\alpha}(\Delta t)=\int_{0}^{\infty} d t J_{\alpha}(t) F_{\mathrm{HCP}}(t-\Delta t)
$$

This signal could be detected similarly to the total induced charge, but weighted with the known driving pulse. The behavior of the Hall current $J_{y}(t)$ observed in Fig. 6 can thus be characterized by a peak at $\Delta t=0$, while a transient current response originating from a pronounced variation with respect to the frequency will not possess this feature. This is confirmed in Fig. 6. 

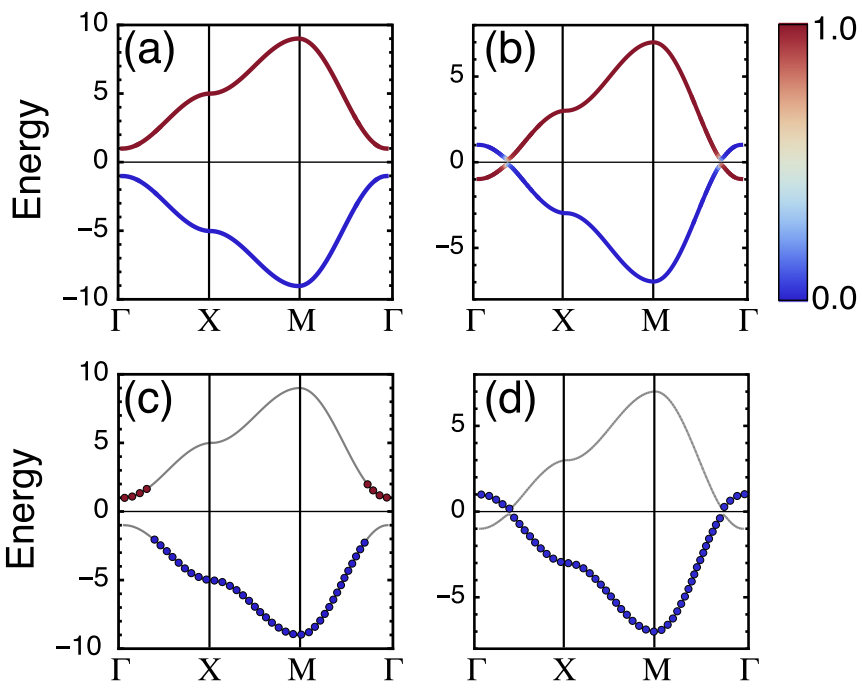

FIG. 7. Band structure of (a) the BI and (b) the TI. The coloring of the lines indicates the orbital weight of the upper band underlying the Hamiltonian (1). Band structure of (c) the BI and (d) the TI, where the dots indicate that the occupation of the respective band after the quench is larger than 0.5. The energy is measured in units of $\left|T_{0}\right|$

\section{TRACING THE QUENCH DYNAMICS: UNITARY TIME EVOLUTION}

Let us now investigate the dynamics of the system after a quench across the phase boundary and the manifestation of this transition in the observables discussed in Sec. III. We focus on the noninteracting case $\gamma=0$ first.

Assuming that the system is initially in equilibrium $(M=$ $\left.M_{\mathrm{TI} / \mathrm{BI}}\right)$ with the lower band completely filled and the upper band empty, the gap parameter is ramped up or down in a time $T_{\mathrm{q}}=5$ (see Sec. II A). This short ramp time corresponds to an almost ideal quench, i.e., the occupations of the postquench bands is given by

$$
f_{n}^{\mathrm{B}}(\mathbf{k})=\left|\left\langle\phi_{\mathbf{k} n}^{\mathrm{B}} \mid \psi_{\mathbf{k} 1}\left(t=T_{\mathrm{q}}\right)\right\rangle\right|^{2} \simeq\left|\left\langle\phi_{\mathbf{k} n}^{\mathrm{B}} \mid \phi_{\mathbf{k} 1}^{\mathrm{A}}\right\rangle\right|^{2} .
$$

The postquench occupation (25) is shown along the standard path in the Brillouin zone of the square lattice in Fig. 7, marked by points where $f_{n}^{\mathrm{B}}(\mathbf{k})>0.5$. In fact, $f_{n}^{\mathrm{B}}(\mathbf{k})$ is close to one for most points.

\section{A. Time-dependent Hall effect}

Examining the structure of the density matrix after the quench, one realizes that coherent superpositions of the two bands play a major role, such that the system is far away from a steady state for which the Hall conductance (7) can be defined. However, to have a measure of the postquench state the system is driven to, we can investigate the response to HCPs as discussed in Sec. III B. The current induced by a HCP polarized along the $x$ direction after performing the quench $M_{\mathrm{BI}} \rightarrow M_{\mathrm{TI}}$ is shown in Fig. 8(a). In comparison to the current response in equilibrium (Fig. 6), the coherent oscillations of the current dominate the hump at times when the electric field of the pulse is strong. The magnitude of these oscillations is considerably larger than in the equilibrium case, showing that
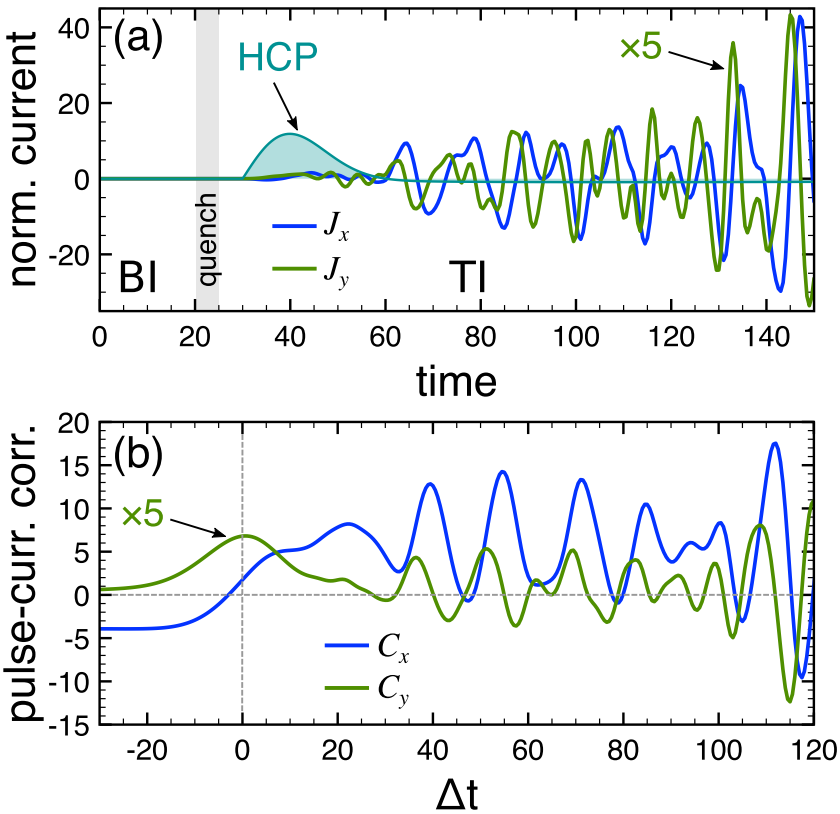

FIG. 8. (a) Time-dependent current induced by a HCP (sketched in the plot) after the quench from the BI state to the TI (illustrated by the shaded background). The current in the $y$ direction has been multiplied by 5 for better visibility. The normalization of the current is consistent with Fig. 6. (b) Corresponding pulse-current correlation functions.

the superposition state after the quench is quite different from the equilibrium TI state.

At first glance, is seems that the current in the $y$ direction is not displaying the behavior discussed in Sec. III B, as the magnitude of the current is quite small even when the electric field reaches its maximum. Nevertheless, the pulse-current correlation function $C_{\alpha}(\Delta t)$ defined in Eq. (24) and presented in Fig. 8(b), exhibits the distinct feature of the QHI: $C_{y}(\Delta t)$ possesses a clear maximum at $\Delta t=0$, which indicates a plateau behavior of the optical conductivity $\sigma_{x y}$ at $\omega=0$ and thus the presence of a nonzero Hall effect. The magnitude of $C_{y}(\Delta t=0)$ is, however, significantly (approximately by a factor of four) reduced with respect to the equilibrium case [Fig. 6(b)]. Hence one would expect a static Hall conductance of about $\sigma_{x y} \simeq 0.25 e^{2} / h$. We also performed analogous simulations for the $M_{\mathrm{TI}} \rightarrow M_{\mathrm{BI}}$ quench and found the emergence of a very small, but finite, Hall effect after the quench.

The coherent superposition present in the postquench state results in an internal dynamics, which might interfere with transient measurements. However, one can expect decoherence effects to diminish these coherences, leading to a mixed steady state. In this case, the static Hall effect yields valuable information on the postquench state, as discussed in the next section.

\section{B. Steady-state conductance}

As can be inferred from Fig. 7, the occupation after the quench reflects the prequench situation. For instance, the complete filling of the lower BI band is preserved when switching to the TI, apart from the avoided crossing points. 

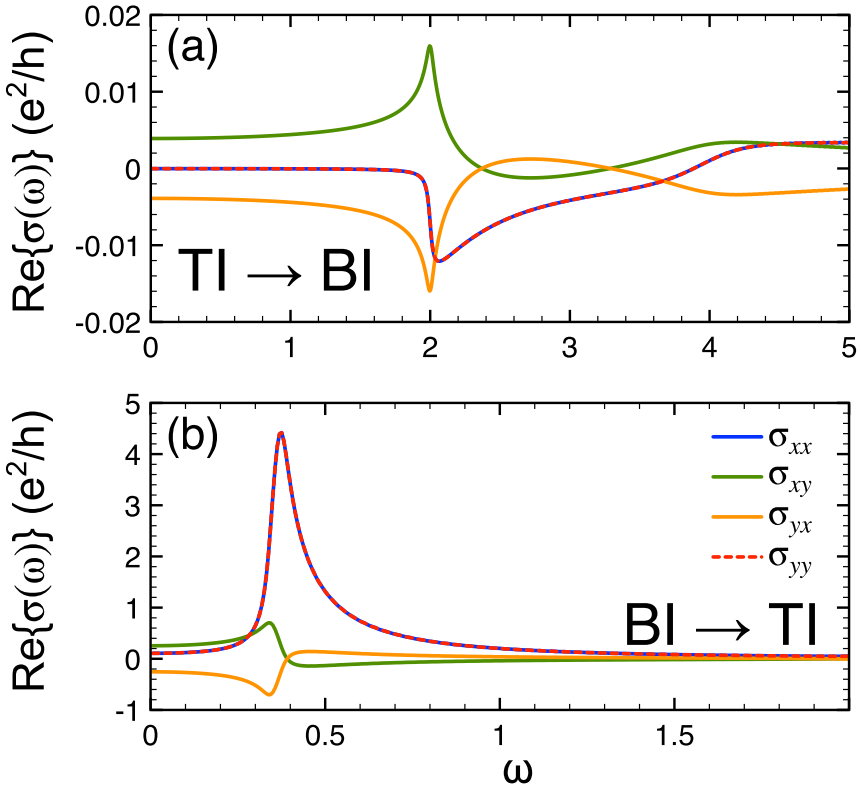

FIG. 9. Optical conductivity of the postquench state $\sigma_{\alpha \beta}^{\mathrm{B}}(\omega)$ according to the definition (26) for (a) the quench $M_{\mathrm{TI}} \rightarrow M_{\mathrm{BI}}$ and (b) $M_{\mathrm{BI}} \rightarrow M_{\mathrm{TI}}$.

This illustrates the conservation of the topological character of the system as the occupation of bands with the same orbital character (which is interchanged at the crossing points in the TI band structure, see Fig. 7) remains constant. Nevertheless, the Hall effect deviates from the equilibrium behavior, which can be seen by evaluating the steady-state optical conductivity in accordance with Ref. [11] by

$$
\begin{aligned}
\sigma_{\alpha \beta}^{\mathrm{B}}(\omega)= & \frac{1}{\omega} \sum_{n} \int d \mathbf{k} f_{n}^{\mathrm{B}}(\mathbf{k}) \int_{0}^{\infty} d t e^{i \omega t} \\
& \times\left\langle\phi_{\mathbf{k} n}^{\mathrm{B}}\left|\left[\hat{j}_{\mathbf{k} \alpha}, \hat{j}_{\mathbf{k} \beta}(t)\right]\right| \phi_{\mathbf{k} n}^{\mathrm{B}}\right\rangle .
\end{aligned}
$$

Here, $\hat{j}_{\mathbf{k} \alpha}=\partial \hat{h}_{\mathrm{el}}^{\mathrm{B}} / \partial k_{\alpha}$ denotes the momentum-resolved current operator. The resulting conductivity is shown in Fig. 9. Note that computing the optical conductivity analogously to Eq. (7) assumes that all off-diagonal elements of the density matrix, which capture the coherent oscillations of the system after the excitation, are zero.

As can be inferred from Fig. 9, the Hall conductance significantly deviates from the equilibrium value. In the $M_{\mathrm{TI}} \rightarrow M_{\mathrm{BI}}$ quench, the system acquires a finite Hall conductance $\sigma_{x y}^{\mathrm{B}}(\omega=$ $0) \simeq 0.0038 e^{2} / h$, while the quench $M_{\mathrm{TI}} \rightarrow M_{\mathrm{BI}}$ leads to $\sigma_{x y}^{\mathrm{B}}(\omega=0) \simeq 0.25 e^{2} / h$. These values are consistent with the time-dependent response discussed in Sec. IV A. Without the unit factor $e^{2} / h$, the latter can be regarded as a nonequilibrium generalization of the Chern number [11]. Note that the concrete numbers depend on both the prequench and the postquench gap parameter $M$.

\section{Transient circular asymmetry}

Let us now proceed to transient properties. As discussed in Sec. III A, the circular asymmetry is a very promising candidate for tracing the dynamics in real time. To find a suitable analog to the equilibrium scenario, we performed test calculations of the population dynamics driven by circularly

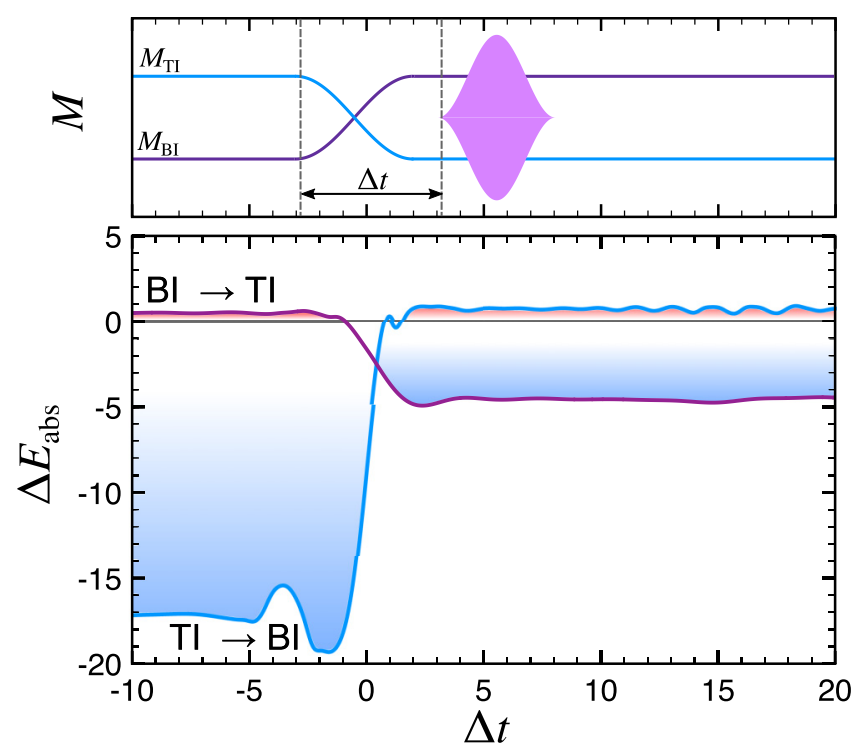

FIG. 10. The circular asymmetry of the absorbed energy $\Delta E_{\mathrm{abs}}$ as a function of the delay $\Delta t$ (bottom) between the quench and the probe pulse (illustrated in the top). The color gradient shading of the curves emphasizes the transition from positive (red) to negative (blue) asymmetry.

polarized pulses (with the same parameters as in Sec. III A and with central frequency $\omega_{0}=\omega_{\text {opt }}$ ) after the system has been quenched. For the case $M_{\mathrm{BI}} \rightarrow M_{\mathrm{TI}}$ one finds that the system is preferably excited by right circular pulses, while a left circular pulse results in a weaker depletion of the postquench lower band. In contrast, circularly polarized pulses applied to the BI after the quench from the TI result in a depletion of the upper band instead (irrespective of the polarization). Furthermore, one observes an oscillatory dependence of the photoexcitation probability after a quench, which originatesanalogously to the current discussed in Sec. IV A-from the coherent superposition of the states belonging to the upper and lower band, respectively.

For these reasons, we propose to utilize the absorbed energy $E_{\text {abs }}$ of the left or right circular probe pulses as a footprint of the topological character in nonequilibrium instead. Importantly, the energy absorption can be measured in experiments directly by placing photon detectors behind the sample. It should be noted that $E_{\mathrm{abs}}$ can also be negative when the system is excited, which corresponds to stimulated emission rather than absorption. This difference to the equilibrium case needs to be taken into account. We thus define a transient generalization of the circular asymmetry by

$$
\Delta E_{\mathrm{abs}}=\left|E_{\mathrm{abs}}^{(+)}\right|-\left|E_{\mathrm{abs}}^{(-)}\right|,
$$

where $E_{\mathrm{abs}}^{( \pm)}$is the energy of the probe pulse, which is absorbed (or emitted) by the system. The time-resolved circular asymmetry signal (27) for both quench scenarios is presented in Fig. 10 as a function of the delay $\Delta t=t_{0}-t_{\mathrm{q}}$ between the starting time of the pulse $\left(t_{0}\right)$ and the time when the system is quenched $\left(t_{\mathrm{q}}\right)$. The absorbed energy is computed by taking the energy difference $E_{\mathrm{abs}}^{( \pm)}=E_{\mathrm{q}+\mathrm{pr}}^{( \pm)}-E_{\mathrm{q}}^{( \pm)}$, where $E_{\mathrm{q}}^{( \pm)}$denotes the total energy of the quenched system in the 
absence of the probe pulse, while $E_{\mathrm{q}+\mathrm{pr}}^{( \pm)}$is the total energy of the quenched and probed target. The energy is measured at a sufficiently large reference time after the quench and the pulse.

Figure 10 demonstrates that the quench dynamics can be traced in the time domain by the circular asymmetry. For the system with $M=M_{\mathrm{BI}}$ before switching, the asymmetry is positive (see Fig. 3). Hence, for $\Delta t<0$ one observes $\Delta E_{\text {abs }}\left(T_{\text {ref }}\right)>0$ in Fig. 10 for the $M_{\mathrm{BI}} \rightarrow M_{\mathrm{TI}}$ case (purple curve). For $\Delta t>0$, the asymmetry assumes negative values (with larger modulus, as well) which, in accordance to Fig. 3, indicates the transition to the TI. The opposite behavior can be observed when switching $M_{\mathrm{TI}} \rightarrow M_{\mathrm{BI}}$ (blue curve). Further features are weakly pronounced coherent oscillations of the asymmetry after the quench to the TI, which originate from the off-diagonal elements of the time-dependent density matrix. The corresponding time scale is determined by the energy difference between the states whose occupation is changed by the quench.

As our time-dependent simulations demonstrate, the timeresolved quench-probe asymmetry signal based on the absorbed energy provides a robust tool to trace the transient dynamics of the system after a quench. It primarily maps out the circular asymmetry of the underlying bands and is less sensitive to the nonequilibrium occupation. These features clearly distinguish the time-resolved asymmetry from the nonequilibrium Hall effect discussed above and render it a powerful complementary tool.

\section{TRACING THE QUENCH DYNAMICS: DISSIPATIVE TIME EVOLUTION}

After having analyzed the unitary dynamics of the system after a quench, we now investigate how the picture changes if EL-PH interactions, as discussed in Sec. II B, are present. Generally, the effect of coupling to the phonon modes is expected to give rise to dissipative dynamics, lowering the energy after the quench excitation. Revisiting Fig. 7 one can expect a qualitatively different behavior for the quench $M_{\mathrm{TI}} \rightarrow$ $M_{\mathrm{BI}}$ as compared to the case $M_{\mathrm{BI}} \rightarrow M_{\mathrm{TI}}$. If the system is quenched from the TI to the BI, the occupation in the upper band [see Fig. 7(a)] is located around the energy minimum at the $\Gamma$ point. Hence, no energy can be extracted from the system after the quench. The effect of the EL-PH coupling is in this case primarily the dephasing of the coherences induced by the quench.

\section{A. Transient dynamics probed by time-resolved photoemission}

We performed numerical simulations of the quench dynamics by solving the master EOM (11) as described in Sec. II C. The parameters are-apart from the EL-PH interaction-the same as in Sec. IV. To understand the time evolution of the band structure and the nonequilibrium occupation, the most convenient quantity to look at is the time-dependent occupation with respect to the postquench basis $f_{n}^{\mathrm{B}}(\mathbf{k} ; t)=$ $\rho_{n n}(\mathbf{k} ; t)$ and-as complementary information-the transient photoelectron spectrum. Time-resolved ARPES (tr-ARPES) has recently become a standard tool for tracing the time evolution in correlated systems [57-60], in parallel with a rapid development of state-of-the-art theoretical descriptions within the NEGF framework [35,36,39]. Modeling the photoemission process by a pump pulse [frequency $\omega$, pulse envelope $F_{\mathrm{pr}}(t)$ ] yields the photocurrent [36]

$$
\begin{aligned}
I(\mathbf{k} ; \omega) \propto & \operatorname{Im} \int_{-\infty}^{\infty} d t \int_{-\infty}^{t} d t^{\prime}\left[F_{\mathrm{pr}}(t)\right]^{*} F_{\mathrm{pr}}\left(t^{\prime}\right) e^{-i \omega\left(t-t^{\prime}\right)} \\
& \times \operatorname{Tr}\left[\mathbf{G}^{<}\left(\mathbf{k} ; t, t^{\prime}\right)\right] .
\end{aligned}
$$

Here, $\mathbf{G}^{<}\left(\mathbf{k} ; t, t^{\prime}\right)$ denotes the lesser Green's function, which we express within the generalized Kadanoff-Baym ansatz as $\mathbf{G}^{<}\left(\mathbf{k} ; t, t^{\prime}\right)=i \mathbf{U}(\mathbf{k} ; t) \boldsymbol{\rho}\left(\mathbf{k} ; t^{\prime}\right)$ with the time-evolution operator corresponding to the single-particle Hamiltonian $\mathbf{h}_{\mathrm{el}}(\mathbf{k} ; t)$. Varying the delay $\Delta t$ between the excitation (the quench at $t_{\mathrm{q}}$ in our case) and the time when the probe pulse is applied, Eq. (28) provides a k- and energy-resolved pump-probe spectrum.

In Fig. 11 we present the time-dependent occupation $f_{n}^{\mathrm{B}}(\mathbf{k} ; t)$ (top row) along with the corresponding tr-ARPES spectra (bottom row) as a function time delay $\Delta t$ for the quench $M_{\mathrm{TI}} \rightarrow M_{\mathrm{BI}}$. In the calculations of the tr-ARPES signals, we used the same parameters for the pulse as in Sec. IV. The short pulse length $T_{\mathrm{p}}=5$ gives rise to the broadening of the spectra in Fig. 11. Since within the weak-coupling regime the steady state does not depend on the strength of the EL-PH coupling, we have chosen the intermediate value $\gamma=0.5$ for a faster dynamics. An analogous relaxation dynamics occurs on longer time scales for smaller $\gamma$. At $\Delta t=-20$, the system is still in equilibrium and the ARPES signal follows the prequench band structure (orange dashed line). The postquench basis is the BI-therefore, the occupation with respect to the BI bands exhibits a hole around the $\Gamma$ point up to the region where the avoided crossing occurs. This demonstrates a different aspect of the topological state: The occupation of the first Brillouin zone with respect to the dominant orbital character of the lower band is not singly connected. Following the time evolution right after the quench $(\Delta t=5)$ we see a similar picture as in Fig. 7: The ARPES spectrum now follows the postquench band structure (solid purple lines), while the upper band is populated around the $\Gamma$ point, whereas the lower band is empty in this region. The population assumes values between zero and one around the crossing region, such that a slight lowering of the total energy by EL-PH relaxation is possible. This effect can be observed for later times $(\Delta t=25)$. The steady state $(\Delta t=120)$, however, shows only a slight blurring of the occupation compared to directly after the quench.

The nonequilibrium dynamics is considerably more pronounced in the quench scenario $M_{\mathrm{BI}} \rightarrow M_{\mathrm{TI}}$, analyzed again in terms of the time-dependent population of the postquench lower band in the tr-ARPES spectra in Fig. 12. Right after the quench $(\Delta t=5)$, the ARPES spectrum closely resembles the BI band structure, apart from a shift to larger energies. However, as the occupation of the lower band shows, the postquench TI band is empty between the $\Gamma$ point and the crossing region, while the upper TI band is populated in this region in the Brillouin zone. It is clear from Fig. 12 that this nonequilibrium occupation does not correspond to an energy minimum as filling the occupation hole in the lower band and a relaxation towards the energy minimum at the crossing points in the upper band result in a lowering of the total electronic energy. These dissipation processes are efficiently mediated by the EL-PH interaction as the time-dependent 

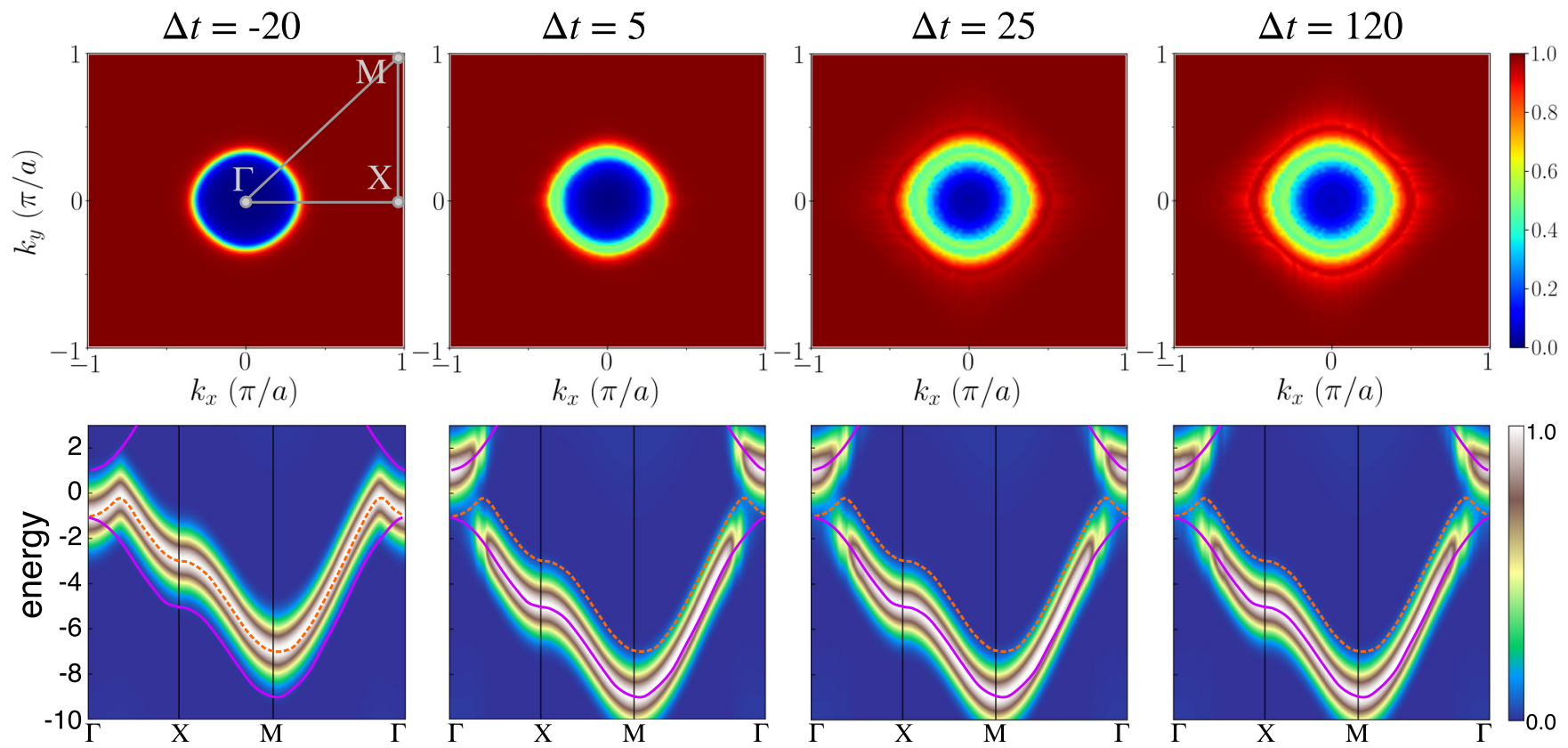

FIG. 11. Top row: time-dependent occupation with respect to the postquench Hamiltonian (defined by $M=M_{\mathrm{BI}}$ ) for different time delays $\Delta t$ relative to the quench time (at $\Delta t=0$ ). Bottom row: corresponding tr-ARPES spectra according to Eq. (28). The full purple lines indicate the post-quench BI band structure, while the dashed orange lines is the lower band of the TI. The EL-PH coupling is set to $\gamma=0.5$.

occupation and the ARPES spectra for later times $(\Delta t=25)$ demonstrate. The steady state reached at $\Delta t=120$ has a peculiar configuration: The occupation hole around the $\Gamma$ point has been filled, while the population of the upper band has relaxed down to the crossing point. Interestingly, from the ARPES spectrum alone one could suspect that the system has fully relaxed to a TI. However, the nonequilibrium occupation, which involves both bands gives rise to a steady-state optical conductivity (Fig. 13), which deviates considerably from the equilibrium behavior (Fig. 5) in its strongly suppressed conductance and Hall conductance. The direct current Hall conductance is reduced to $\sigma_{x y} \simeq 0.003 e^{2} / h$ for the quench to the $\mathrm{BI}$, while we find $\sigma_{x y} \simeq 0.05 e^{2} / h$ in the $M_{\mathrm{BI}} \rightarrow M_{\mathrm{TI}}$ scenario. Note that the value of the Hall conductance in the TI final state is considerably smaller than in the quench scenario without EL-PH interaction (Fig. 9). This can be explained by the distinct occupation in the postquench steady state in the presence of the EL-PH coupling. The integer Hall conductance $\sigma_{x y}=e^{2} / h$ originates from interband transitions in the crossing region of the upper and lower band. Exactly
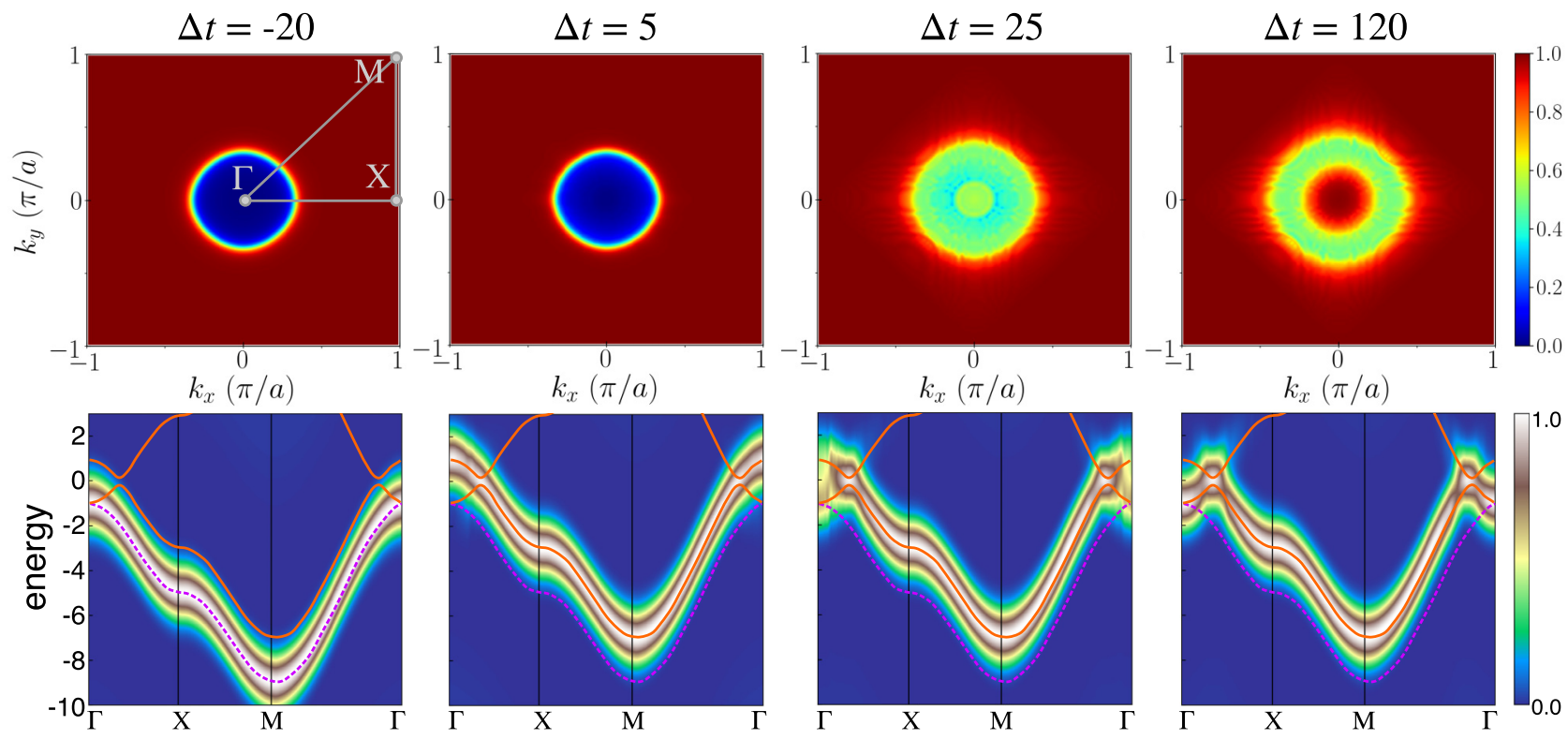

FIG. 12. Top row: Time-dependent occupation with respect to the postquench Hamiltonian (defined by $M=M_{\mathrm{TI}}$ ) for different time delays $\Delta t$ as in Fig. 11. Bottom row: tr-ARPES spectra according to Eq. (28). The full orange lines indicate the postquench TI band structure, while the dashed purple line represents the lower band of the BI. The EL-PH coupling is set to $\gamma=0.5$. 

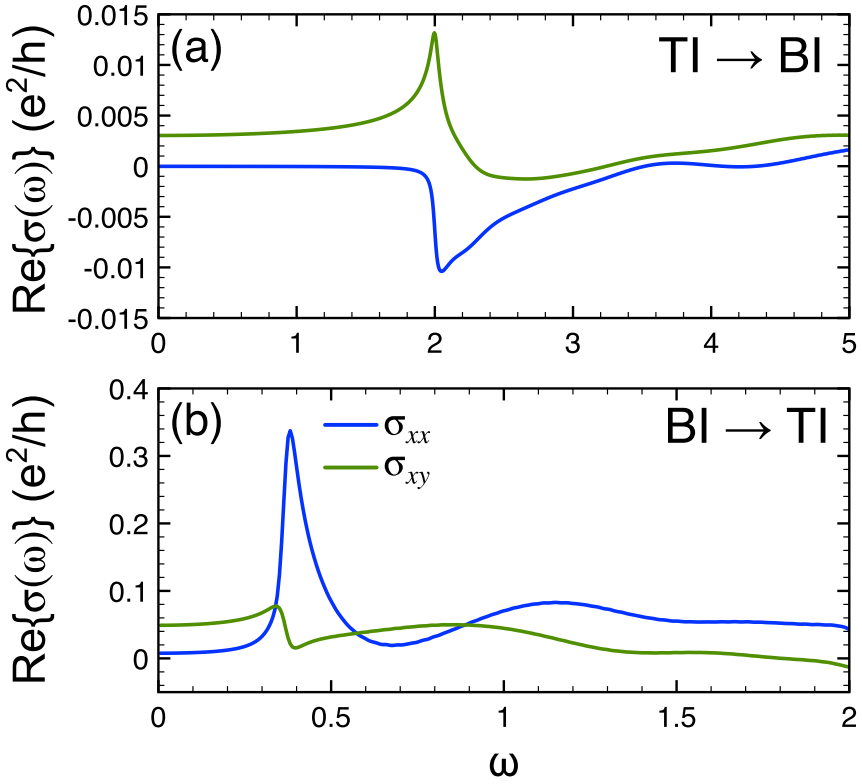

FIG. 13. Real part of the nonequilibrium optical conductivity of the postquench steady-state reached in the presence of EL-PH interactions, for (a) the transition $M_{\mathrm{TI}} \rightarrow M_{\mathrm{BI}}$, and (b) the switching $M_{\mathrm{BI}} \rightarrow M_{\mathrm{TI}}$.

those transitions are strongly suppressed in the steady state of Fig. 12, as the occupation in the lower band in the crossing region is depleted, while the available states in the upper band are mostly occupied.

\section{B. Transient circular asymmetry}

Let us now investigate if the transition from the topologically trivial $\mathrm{BI}$ to the $\mathrm{QHI}$ or vice versa can be traced in the time domain by circularly polarized pulses in an analogous fashion as for the dissipationless case discussed in Sec. IV C. We employ the same scheme: a left or right circularly polarized pulse photoexcites the system before, during or after the quench. The energy absorbed by the pulse and, in particular, the left-right asymmetry should then reveal the character of the steady state or transient state. We have applied this recipe, using the definition (27), and present the respective energy absorption asymmetry in Fig. 14 for two representative cases of the EL-PH interaction: (i) weak coupling $\gamma=0.1$ [Figs. 14(a)-14(b)] and moderate coupling strength $\gamma=0.3$ [Figs. 14(c)-14(d)]. We represent the absorption asymmetry as a function of the quench-pulse delay $\Delta t$ as in Fig. 10.

For $\gamma=0.1$, Fig. 14(a) shows a transition $M_{\mathrm{BI}} \rightarrow M_{\mathrm{TI}}$ similarly to the unitary case. The asymmetry $\Delta E_{\text {abs }}$ changes from positive values (with small magnitude) to negative, indicating the switch from BI to TI. On the other hand, the quench $M_{\mathrm{TI}} \rightarrow M_{\mathrm{BI}}$ [Fig. 14(b)] is accompanied by a switch of $\Delta E_{\text {abs }}$ from negative to positive values (with strongly reduced magnitude). Generally, the scheme for tracing the quench dynamics works very well for the dissipative case with weak EL-PH interaction.

Turning to stronger EL-PH couplings, the picture slightly changes. For the quench $M_{\mathrm{BI}} \rightarrow M_{\mathrm{TI}}$ [Fig. 14(c)], one can observe the asymmetry $\Delta E_{\mathrm{abs}}$ switching from small positive
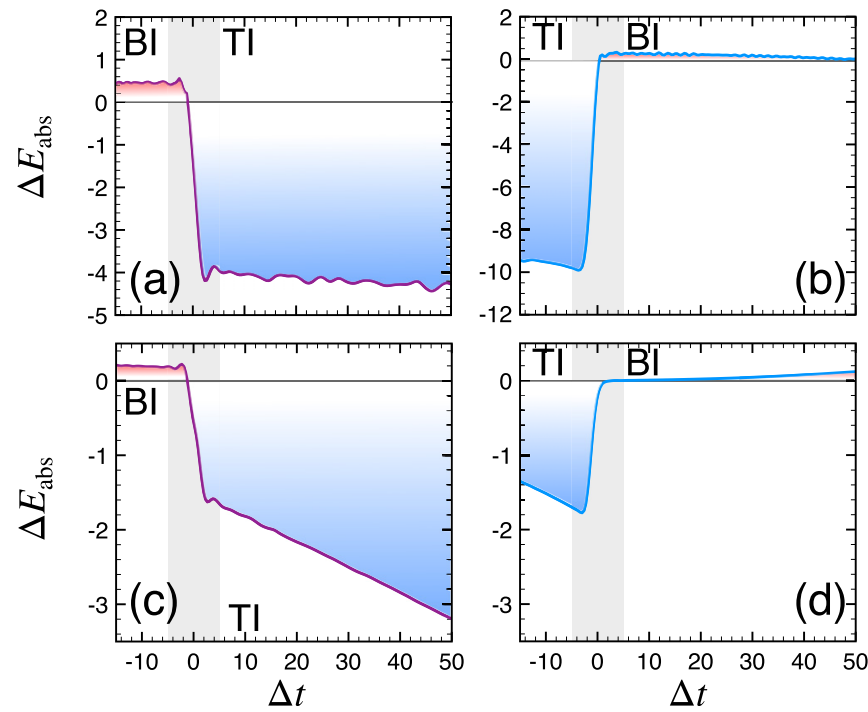

FIG. 14. Asymmetry of the absorbed energy $\Delta E_{\text {abs }}$ as a function of the delay $\Delta t$ between the quench and the probe pulse for (a)-(b) weak EL-PH coupling $\gamma=0.1$, and (c)-(d) moderate EL-PH coupling $\gamma=0.3$. The color gradient filling of the curves is analogous to Fig. 10. The gray shaded area indicates the time when the system is switched from BI to TI (left panels) or TI to BI (right panels), respectively.

values to the negative region across the quench. The almost linear decrease of $\Delta E_{\mathrm{abs}}$ towards larger $\Delta t$ is attributed to the EL-PH induced relaxation after the pulse (see Fig. 12). One can readily check that the photoexcitation probability due to the specific pulse is larger in the postquench relaxed steady state than right after the quench, because more states are occupied in the energy window defined by the pulse frequency. Therefore, the absorption of the left-circular probe pulse becomes more efficient as $\Delta t$ increases, until the steady state is reached (at $\Delta t \sim 200)$. Generally, the dependence of $\Delta E_{\mathrm{abs}}$ on $\Delta t$ is much more pronounced in the TI phase, since the influence of the EL-PH coupling (as discussed in Sec. V A) is stronger.

\section{CONCLUSIONS}

We have studied the quench dynamics of the MDM as a generic model for two-dimensional topological insulators with a special emphasis on how the nonequilibrium and transient properties are reflected in experimentally accessible quantities. We have focused on two promising observables, which reveal the topological character of the system: the steady-state and time-dependent Hall effect and the asymmetry of photoexcitation with respect to left or right circularly polarized pulses. Based on a realistic model for two-dimensional topological insulators we have defined suitable probe-pulse shapes by considering the equilibrium model, both for the dissipationless case and including electron-phonon interactions. We then applied these optimized pulses to trace the nonequilibrium dynamics after a quench. Both the time-dependent Hall effect and the circular dichroism of the absorbed energy provide valuable information on the system. While the Hall current can deliver important insights into the steady state, it turns out to be less suitable for the analysis of transient states. This is due 
to the coherent superpositions in the system, which give rise to an intrinsic transient dynamics. The circular asymmetry of the absorbed energy, on the other hand, is much less sensitive to these effects, since it is based on the occupation of the bands only. The latter approach is thus particularly well suited for the study of the switching process between different phases.

In the presence of electron-phonon coupling, the quench dynamics can significantly differ from the dissipationless case, provided the energy of the system is reduced by scattering from phonons. We analyzed these effects in terms of the time-resolved ARPES spectra. We investigated the steadystate properties by the nonequilibrium Hall conductance and, finally, analyzed the quench dynamics in terms of the circular asymmetry. As an important point for the potential experimental realization, we have demonstrated the robustness of our proposed transient measurement in the presence of weak to moderate electron-phonon coupling. The transient circular dichroism is therefore a promising tool, which provides insights into the little explored field of topological phases in nonequilibrium.

\section{ACKNOWLEDGMENTS}

The calculations have been performed on the Beo04 cluster at the University of Fribourg. This work has been supported by the Swiss National Science Foundation through NCCR MARVEL and ERC Consolidator Grant No. 724103. We thank Markus Schmitt and Stefan Kehrein for fruitful discussions.

\section{APPENDIX: NUMERICAL SOLUTION OF THE MASTER EQUATION}

For a stable numerical solution of a master equation of the type of Eq. (11) we adapt a method for propagating NEGFs in time [48]. The interval $\left[0, t_{\max }\right]$ is discretized into an equidistant grid $t_{n}=n \Delta t$. In order to perform the step $\rho\left(t_{n}\right) \rightarrow \rho\left(t_{n+1}\right)$, we separate the unitary time evolution from the scattering term by the ansatz

$$
\begin{aligned}
\rho\left(t_{n}+\tau\right) & =\mathbf{U}\left(t_{n}+\tau, t_{n}\right) \tilde{\boldsymbol{\rho}}_{n}(\tau) \mathbf{U}^{\dagger}\left(t_{n}+\tau, t_{n}\right) \\
& \equiv \mathbf{U}_{n}(\tau) \widetilde{\boldsymbol{\rho}}_{n}(\tau) \mathbf{U}_{n}^{\dagger}(\tau), \tau \in[0, \Delta t] .
\end{aligned}
$$

Here, $\mathbf{U}\left(t_{n}+\tau, t_{n}\right)$ denotes the time-evolution operator, which we approximate by $\mathbf{U}_{n}(\tau) \approx \exp \left[-i \tau \mathbf{h}_{\mathrm{el}}\left(t_{n}+\Delta t / 2\right)\right]$. Inserting Eq. (A1) into the EOM (11) then yields

$$
\begin{aligned}
\rho\left(t_{n+1}\right)= & \mathbf{U}_{n}(\Delta t) \rho\left(t_{n}\right) \mathbf{U}_{n}^{\dagger}(\Delta t) \\
& +\int_{0}^{\Delta t} d \tau \mathbf{U}_{n}^{\dagger}(\tau-\Delta t) \mathbf{I}\left(t_{n}+\tau\right) \mathbf{U}_{n}(\tau-\Delta t) .
\end{aligned}
$$

Apart from the approximation to the time-evolution operator $\mathbf{U}_{n}(\tau)$, Eq. (A2) is still exact. A simple and numerically stable propagation scheme is obtained by approximating $\mathbf{I}\left(t_{n}+\tau\right) \approx$ $\mathbf{I}\left(t_{n}+\Delta t / 2\right) \equiv \mathbf{I}_{n+1 / 2}$. Using the Baker-Hausdorff formula, the time step (A2) can be expressed as

$$
\begin{aligned}
\rho\left(t_{n+1}\right)= & \mathbf{U}_{n}(\Delta t) \rho\left(t_{n}\right) \mathbf{U}_{n}^{\dagger}(\Delta t) \\
& +i \Delta t \sum_{k=0}^{p-1} \frac{(-i \Delta t)^{k}}{k !} \mathbf{C}_{n}^{(k)}+O\left[(\Delta t)^{p+1}\right]
\end{aligned}
$$

with $\mathbf{C}_{n}^{(0)}=\mathbf{I}_{n+1 / 2}$ and $\mathbf{C}_{n}^{(k+1)}=\left[\mathbf{h}_{\mathrm{el}}\left(t_{n}+\Delta t / 2\right), \mathbf{C}_{n}^{(k)}\right]$. In practice, we truncate Eq. (A3) after the fourth order $(p=4)$. The half-step scattering term $\mathbf{I}_{n+1 / 2}$ is obtained by fourth-order polynomial interpolation using $\mathbf{I}_{n+1-k}, k=0, \ldots, 3$. This requires knowing the scattering term at the next time step, $\mathbf{I}_{n+1}$, which is a function of the yet unknown density matrix $\boldsymbol{\rho}\left(t_{n+1}\right)$. Therefore, we employ a predictor-corrector scheme where $\mathbf{I}_{n+1 / 2}$ is first estimated by third-order polynomial extrapolation, allowing to compute $\rho\left(t_{n+1}\right)$ and thus obtain $\mathbf{I}_{n+1}$. The latter two steps are then iterated at each time step until $\rho\left(t_{n+1}\right)$ is converged.
[1] M. Z. Hasan and C. L. Kane, Rev. Mod. Phys. 82, 3045 (2010).

[2] J. E. Moore, Nature (London) 464, 194 (2010).

[3] D. J. Thouless, M. Kohmoto, M. P. Nightingale, and M. den Nijs, Phys. Rev. Lett. 49, 405 (1982).

[4] Z. Wang and S.-C. Zhang, Phys. Rev. X 2, 031008 (2012).

[5] J. C. Budich, B. Trauzettel, and G. Sangiovanni, Phys. Rev. B 87, 235104 (2013).

[6] A. Amaricci, J. C. Budich, M. Capone, B. Trauzettel, and G. Sangiovanni, Phys. Rev. Lett. 114, 185701 (2015).

[7] P. Kumar, T. Mertz, and W. Hofstetter, Phys. Rev. B 94, 115161 (2016).

[8] Z. Wang, X.-L. Qi, and S.-C. Zhang, Phys. Rev. Lett. 105, 256803 (2010).

[9] J. Fröhlich and P. Werner, Europhys. Lett. 101, 47007 (2013).

[10] M. D. Caio, N. R. Cooper, and M. J. Bhaseen, Phys. Rev. Lett. 115, 236403 (2015).

[11] P. Wang, M. Schmitt, and S. Kehrein, Phys. Rev. B 93, 085134 (2016).

[12] F. N. Ünal, E. J. Mueller, and M. O. Oktel, Phys. Rev. A 94, 053604 (2016).
[13] A. A. Patel, S. Sharma, and A. Dutta, Eur. Phys. J. B 86, 367 (2013).

[14] M. Schmitt and P. Wang, Phys. Rev. B 96, 054306 (2017).

[15] D. T. Tran, A. Dauphin, A. G. Grushin, P. Zoller, and N. Goldman, Sci. Adv 3, e1701207 (2017).

[16] X.-L. Qi, T. L. Hughes, and S.-C. Zhang, Phys. Rev. B 78, 195424 (2008).

[17] C.-X. Liu, X.-L. Qi, X. Dai, Z. Fang, and S.-C. Zhang, Phys. Rev. Lett. 101, 146802 (2008).

[18] B. A. Bernevig, T. L. Hughes, and S.-C. Zhang, Science 314, 1757 (2006).

[19] M. Heyl and J. C. Budich, arXiv:1705.08980 [cond-mat, physics:quant-ph].

[20] M. Eckstein and P. Werner, Phys. Rev. Lett. 110, 126401 (2013).

[21] D. Golež, L. Boehnke, H. U. R. Strand, M. Eckstein, and P. Werner, Phys. Rev. Lett. 118, 246402 (2017).

[22] N. H. Lindner, G. Refael, and V. Galitski, Nature Phys. 7, 490 (2011).

[23] L. D'Alessio and M. Rigol, Nat. Commun. 6, 8336 (2015). 
[24] Y. Hu, P. Zoller, and J. C. Budich, Phys. Rev. Lett. 117, 126803 (2016).

[25] S. Wolff, A. Sheikhan, and C. Kollath, Phys. Rev. A 94, 043609 (2016).

[26] H. Dehghani, T. Oka, and A. Mitra, Phys. Rev. B 91, 155422 (2015).

[27] H. Dehghani and A. Mitra, Phys. Rev. B 92, 165111 (2015).

[28] R. van Leeuwen, Phys. Rev. B 69, 115110 (2004).

[29] T. Ouyang and M. Hu, J. Appl. Phys. 117, 245101 (2015).

[30] S. Giraud and R. Egger, Phys. Rev. B 83, 245322 (2011).

[31] S. Giraud, A. Kundu, and R. Egger, Phys. Rev. B 85, 035441 (2012).

[32] M. V. Costache, I. Neumann, J. F. Sierra, V. Marinova, M. M. Gospodinov, S. Roche, and S. O. Valenzuela, Phys. Rev. Lett. 112, 086601 (2014).

[33] R. C. Hatch, M. Bianchi, D. Guan, S. Bao, J. Mi, B. B. Iversen, L. Nilsson, L. Hornekær, and P. Hofmann, Phys. Rev. B 83, 241303 (2011).

[34] Z.-H. Pan, A. V. Fedorov, D. Gardner, Y. S. Lee, S. Chu, and T. Valla, Phys. Rev. Lett. 108, 187001 (2012).

[35] M. Sentef, A. F. Kemper, B. Moritz, J. K. Freericks, Z.-X. Shen, and T. P. Devereaux, Phys. Rev. X 3, 041033 (2013).

[36] A. F. Kemper, M. Sentef, B. Moritz, C. C. Kao, Z. X. Shen, J. K. Freericks, and T. P. Devereaux, Phys. Rev. B 87, 235139 (2013).

[37] Y. Murakami, P. Werner, N. Tsuji, and H. Aoki, Phys. Rev. B 91, 045128 (2015).

[38] N. Säkkinen, Y. Peng, H. Appel, and R. v. Leeuwen, J. Chem. Phys. 143, 234102 (2015).

[39] Y. Murakami, P. Werner, N. Tsuji, and H. Aoki, Phys. Rev. B 94, 115126 (2016).

[40] M. Schüler, J. Berakdar, and Y. Pavlyukh, Phys. Rev. B 93, 054303 (2016).

[41] R. Tuovinen, N. Säkkinen, D. Karlsson, G. Stefanucci, and R. van Leeuwen, Phys. Rev. B 93, 214301 (2016).

[42] H.-P. Breuer and F. Petruccione, The Theory of Open Quantum Systems (Oxford University Press, Oxford, 2002).
[43] S. Usenko, M. Schüler, A. Azima, M. Jakob, L. L. Lazzarino, Y. Pavlyukh, A. Przystawik, M. Drescher, T. Laarmann, and J. Berakdar, New J. Phys. 18, 113055 (2016).

[44] R. Rosati, R. C. Iotti, F. Dolcini, and F. Rossi, Phys. Rev. B 90 , 125140 (2014).

[45] R. Rosati, F. Dolcini, and F. Rossi, Phys. Rev. B 92, 235423 (2015).

[46] N. Schlünzen and M. Bonitz, Contrib. Plasma Phys. 56, 5 (2016).

[47] D. C. Langreth and P. Nordlander, Phys. Rev. B 43, 2541 (1991).

[48] A. Stan, N. E. Dahlen, and R. v. Leeuwen, J. Chem. Phys. 130, 224101 (2009).

[49] C. Hwang, C.-H. Park, D. A. Siegel, A. V. Fedorov, S. G. Louie, and A. Lanzara, Phys. Rev. B 84, 125422 (2011).

[50] Y. Liu, G. Bian, T. Miller, and T.-C. Chiang, Phys. Rev. Lett. 107, 166803 (2011).

[51] L. Duca, T. Li, M. Reitter, I. Bloch, M. Schleier-Smith, and U. Schneider, Science 347, 288 (2015).

[52] Z. Wu, L. Zhang, W. Sun, X.-T. Xu, B.-Z. Wang, S.-C. Ji, Y. Deng, S. Chen, X.-J. Liu, and J.-W. Pan, Science 354, 83 (2016).

[53] A. S. Moskalenko, Z.-G. Zhu, and J. Berakdar, Phys. Rep. 672, 1 (2017).

[54] A. S. Moskalenko, A. Matos-Abiague, and J. Berakdar, Phys. Rev. B 74, 161303 (2006).

[55] D. You, D. R. Dykaar, R. R. Jones, and P. H. Bucksbaum, Opt. Lett. 18, 290 (1993).

[56] R. R. Jones, D. You, and P. H. Bucksbaum, Phys. Rev. Lett. 70, 1236 (1993).

[57] F. Schmitt, P. S. Kirchmann, U. Bovensiepen, R. G. Moore, L. Rettig, M. Krenz, J.-H. Chu, N. Ru, L. Perfetti, D. H. Lu, M. Wolf, I. R. Fisher, and Z.-X. Shen, Science 321, 1649 (2008).

[58] J. Graf, C. Jozwiak, C. L. Smallwood, H. Eisaki, R. A. Kaindl, D.-H. Lee, and A. Lanzara, Nat. Phys. 7, 805 (2011).

[59] U. Bovensiepen and P. Kirchmann, Laser \& Photonics Reviews 6, 589 (2012).

[60] C. L. Smallwood, J. P. Hinton, C. Jozwiak, W. Zhang, J. D. Koralek, H. Eisaki, D.-H. Lee, J. Orenstein, and A. Lanzara, Science 336, 1137 (2012). 